\title{
BMJ Open Transition in acquired brain injury youth (TrABI-Y): a systematic literature review protocol
}

\author{
Jérôme Gauvin-Lepage (D) ,, Julie Farthing, ${ }^{1,3}$ Shana Bissonnette, ${ }^{1,4}$ \\ Louise Koclas, ${ }^{5}$ Magdalena Jaworski, ${ }^{6}$ Josée Larochelle, ${ }^{6}$ \\ Anne-Sophie St-Pierre-Clément ${ }^{7}$
}

To cite: Gauvin-Lepage J, Farthing J, Bissonnette S, et al. Transition in acquired brain injury youth (TrABI-Y): a systematic literature review protocol. BMJ Open 2019;9:e027384. doi:10.1136/ bmjopen-2018-027384

- Prepublication history and additional material for this paper are available online. To view these files, please visit the journal online (http://dx.doi. org/10.1136/bmjopen-2018027384).

Received 19 0ctober 2018 Revised 19 August 2019 Accepted 23 September 2019

Deck for updates

(c) Author(s) (or their employer(s)) 2019. Re-use permitted under CC BY-NC. No commercial re-use. See rights and permissions. Published by BMJ.

For numbered affiliations see end of article.

Correspondence to Dr Jérôme Gauvin-Lepage; jerome.gauvin-lepage@ umontreal.ca

\section{ABSTRACT}

Introduction Acquired brain injury $(\mathrm{ABI})$ in paediatrics refers to children born with a neurological deficit, which will lead to a chronic neurological disorder. As advances in medical paediatric health progress, we are seeing these $\mathrm{ABI}$ youth transitioning into adult healthcare services while also going through different life events. Despite the growing number of young adult patients, access to transition programmes to facilitate the transition process is still limited and evidence on the effectiveness of these programmes is inconclusive. The purpose of this paper is to provide the protocol for an upcoming systematic literature review on this important issue.

Methods and analysis The start of this systematic literature review is planned for 1 October 2019 and will end on 31 December 2021. According to the PICO framework developed, the Population and the Problem of interest $(P)$ will include children, adolescents and young adults (0-18 years) diagnosed with $\mathrm{ABI}$. The selected articles will have to involve an Intervention (I) relating to the healthcare of this population or life transitions from paediatric to adult-oriented care. Studies will be included if a Comparator (C) intervention was used. The expected Outcomes $(0)$ will have to report quantitative or qualitative health-related outcomes post-transition. This comprehensive search of peer-reviewed literature will include articles published between 2010 and 2020. The databases to be searched include Medline, All EBM Reviews, Embase, PsycINF0 and CINAHL. The selected articles will be appraised using the Mixed Methods Appraisal Tool. A synthesis of the findings will be drafted to identify the effectiveness of available transition programmes as well as predictors, factors and determinants involved in the transition process.

Ethics and dissemination This project is not associated with direct individuals. The dissemination plan includes strategies such as using this systematic literature review to develop a research project on transition that will be published.

\section{INTRODUCTION}

Transition leads to multiple simultaneous changes that require great adaptability, especially in youth with acquired brain injury (ABI). ABI in paediatrics refers to children born with a neurological condition, which

\section{Strengths and limitations of this study}

- This study will contribute to a better understanding of the current state of knowledge regarding the transitions of care and life processes of acquired brain injury (ABI) youth by identifying the effectiveness of available transition programmes for these individuals as well as predictors, factors and determinants involved.

- This study will also guide decision makers in proposing innovative and integrated solutions for the transitions of care and life processes for the $\mathrm{ABI}$ youth population.

- The Mixed Methods Appraisal Tool will be used to ascertain the quality of data obtained by each paper for the primary and secondary outcomes in an evidentiary table.

- Only electronic databases will be searched; hence, publication bias cannot be ruled out.

- The literature review to be conducted will not be a meta-analysis given that the studies will most probably differ significantly with regard to design, purposes and outcomes.

results in a chronic neurological disorder. ${ }^{1}$ These disorders can stem from a number of neurological conditions, such as cerebral palsy or Down syndrome. According to the Society for Adolescent Medicine, the transition of care process is defined as: 'a purposeful, planned process that addresses the medical, psychosocial, and educational/vocational needs of adolescents and young adults with chronic physical and medical conditions as they move from child-centred to adult-oriented health care systems'. ${ }^{2}$ In other words, transition refers to a planned and complex process, rather than an event or a single step at a point in time, that ensures the continuity and coordination of health and life care as youth transfers within and across healthcare and public facilities. ${ }^{3-7}$ The definition used in this literature review protocol was broadened to include all types of transitions that can 
occur during the life of a child with an ABI, such as transitioning through school (eg, from high school to college), work, rehabilitation centres, home care and adult healthcare centres.

The literature highlights the need to provide effective transition services in the healthcare field for $\mathrm{ABI}$ youth, ${ }^{89}$ as these are essential for future quality of life. Indeed, advances in paediatric healthcare have led to an increase in survival rates of children with complex chronic neurological conditions and thus a rise in young adults with previously unseen conditions admitted and treated in adult healthcare centres. ${ }^{910}$ Providing sufficient preparation prior to the transition from paediatric to adult care services is crucial, as patients often feel anxious and concerned at the knowledge of being transited. ${ }^{31112}$ Differences between paediatric and adult healthcare settings can lead to consequences for the transitioning youth population, as paediatric healthcare providers tend to ignore the growing independence of adolescents while adult care providers encourage their patients to take responsibility for their care and health. ${ }^{3}{ }^{13-16}$ Several studies have stressed the positive correlation between lack of readiness for transition and deterioration in the health status of young people with ABI. ${ }^{17-19}$ As a result, adolescents and young adults transitioning to adult care facilities often feel unprepared, which leads to lower rates of follow-up appointments, attendance and medication compliance. ${ }^{30}$ It is important for adolescents and young adults to learn self-care and self-empowerment, as well as to develop effective, lasting partnerships with the healthcare providers involved in their care. ${ }^{89}$

A range of approaches and strategies, including structured transitioning programmes, have been developed and implemented to improve youth's health outcomes. ${ }^{321-24}$ The mechanism of action of most transitioning programmes often includes six core elements to facilitate transition preparation, transfer of care and integration into adult care centres, which include establishing a policy, tracking progress, administering transition readiness assessments, planning for adult care, transferring and integrating into an adult practice. ${ }^{325} 26$ Transition programmes have been shown to help ABI youth maintain healthy habits in adulthood as well as ensure compliance with their treatment. ${ }^{89}$ These benefits and improved health outcomes have also been noted in other youth population with chronic illnesses, especially for patients with cystic fibrosis and diabetes, following a structured multidisciplinary transition programme. ${ }^{3}$ However, the availability of these transition programmes is still limited and evidence on their effectiveness is inconclusive, which may be the result of wide variations in the structure and delivery of these programmes. ${ }^{3}$ 17-19 2728

Hence, a better understanding of the current state of knowledge regarding transitions of care and life processes of ABI youth would allow clinicians and researchers to better identify the effectiveness of available transition programmes for this population. These results could further be used as a guide for national and international decision makers interested in proposing innovative and integrated solutions for the transitions of care and life processes for the ABI youth population.

In short, the purpose of this paper is to provide a protocol for an upcoming systematic literature review on the topic of transitions of care and life processes for children, adolescents and young adults with ABI from 2010. This review will attempt to address two research questions: (1) What is the effectiveness of transition programmes available for children, adolescents and young adults with ABI? and (2) What are the predictors, factors and determinants involved in the transition process of this population?

\section{METHODS AND ANALYSIS}

\section{Patient and public involvement}

Patients, families and/or the public were not involved in the design of this study or in the development of the research goal and objectives.

\section{Search strategy}

The start of this systematic literature review is planned for 1 October 2019 and will end on 31 December 2021. The objective is to review the state of current knowledge regarding transition programmes available for youth with ABI. A mixed-methods systematic review will be conducted in accordance with the PRISMA-P guidelines. ${ }^{29}$

A comprehensive search strategy for finding relevant peer-reviewed literature will be developed. Sources of peer-reviewed literature to be searched will include the databases Ovid Medline, Ovid All EBM Reviews, Ovid Embase, Ovid PsycINFO and EBSCO Cumulative Index of Nursing and Allied Health (CINAHL) (see online supplementary appendix 1). The criterion for inclusion will be publication dates between 2010 and 2020. Articles published before 2010 will not be considered, as evidence regarding the research topic may be outdated. Search terms will be: ABI, cerebral palsy, Down syndrome, brain paralysis, central paralysis, encephalopathia infantilis, little disease, spastic diplegia, transition, paediatric, child, adolescent, families and parents and may be present in the title, abstract and keywords of publications. Furthermore, articles' bibliographies and reference lists will be evaluated for other potentially relevant articles. Restrictions will include articles available in English or French and related to human studies. This systematic literature review will be completed in collaboration with a librarian of Sainte-Justine University Health Centre with special training and skills in literature searches.

\section{Inclusion criteria}

A PICO framework was developed to guide this search. Thus, the inclusion criteria for this upcoming systematic review will include:

- Articles that refer to the eligible Population and Problem of interest $(\mathrm{P})$, which comprises children, adolescents and young adults (0-18 years) diagnosed 
with the following ABI conditions: cerebral palsy, Down syndrome, encephalopathia infantilis, little disease and spastic diplegia. In other words, any condition that meets the ABI definition provided in this protocol will be included in this systematic literature review.

- Any Intervention (I) relating to the healthcare of this population or life transitions from paediatric to adult-oriented care. These interventions can include approaches, strategies and/or structured transition programmes that facilitated the transition from school, work, rehabilitation centres, home care and/ or adult healthcare centres. ${ }^{3} 14212230$ Examples of interventions include: transition education/training, self-management training, care coordination, shared care, introduction to the adult team prior to transfer, move from high school to college and so on. ${ }^{25}$

- Studies that involve a Comparator (C) intervention. These can refer to usual care, transfer letter alone and scheduling an adult visit. Pre-post testing will also be acceptable. $^{25}$

- Any quantitative or qualitative health-related Outcomes $(\mathrm{O})$ post-transition according to age groups, ABI health condition or to types of transition processes. Expected primary outcome measures may include mean of time to transfer, measures of disease control, proportion of patients transferred, percentage of mortality, frequency of emergency room use, patient-reported outcomes, views, experiences and perceptions of the transition. ${ }^{325}$ In addition, as secondary outcome measures, the predictors, factors and determinants involved in the transition process of this population will also be of interest.

- Quantitative (eg, randomised control trials, non-randomised controlled trials and cross-sectional studies), qualitative (eg, grounded theory, phenomenology and ethnography) and mixed-methods designs will be included in this review. The studies can be of any duration.

\section{Exclusion criteria}

The exclusion criteria for this systematic literature review will include: $:^{25}$

- Concerning types of participants, studies referring to youth (0-18 years) who suffered non-congenital neurotrauma (ie, brain injury, stroke, spinal cord injury and meningitis) will be excluded. Studies that include participants older than the target age range are eligible, as long as they include participants in the target age range as well.

- Studies that had no specific intervention will not be included. Studies that were only observational will be excluded as well.

- If no comparator was used or if the comparison was unrelated to the intervention, the studies will not be eligible.

- If no outcomes or specific quantitative or qualitative outcomes are reported, the studies will be excluded.
- Regarding types of studies, all grey literature will be excluded, except for published abstracts of conference proceedings that have been peer reviewed.

\section{Selection of articles}

Two review authors will independently screen the search outputs for potentially eligible articles (authors SB and $\mathrm{JF}$ ). The two independent reviewers will screen the titles and abstracts of the publications for relevance, and all studies that do not meet inclusion criteria will be automatically removed. Subsequently, full texts of potentially eligible articles will be appraised independently by the two authors to assess them for eligibility based on the study inclusion criteria. A third review author (principal author JGL) will assess the articles selected before their final approval for the systematic literature review. All reviewers will need to agree on the final articles to be included, with disagreements to be resolved through discussion and mutual consensus.

\section{Screening and data extraction}

For the two reviewers (authors SB and JF) to be able to independently extract data regarding the eligible studies, a composite evidentiary table will be constructed. This evidentiary table will include: the internal identification number, authors, year, design type, aim of the study, sample size, transition programme characteristics, clinical variables assessed, outcome measures, use and application in children and adolescents with $\mathrm{ABI}$, predictors, factors and/or determinants, if any, results/conclusions as well as strengths and limitations (eg, bias).

\section{Quality assessment}

To ensure quality in the systematic literature review, articles selected will be appraised by two authors (authors SB and JF) using the Mixed Methods Appraisal Tool (MMAT), V.2018. ${ }^{31}$ The MMAT is a validated and reliable checklist developed to assess the methodological quality of quantitative, qualitative and mixed-methods studies included in systematic reviews of mixed studies. ${ }^{32}$ Each study will be judged according to its methodology (ie, qualitative, quantitative, randomised controlled trials, quantitative non-randomised, quantitative descriptive and mixed methods). Then, the five criteria provided in the MMAT for each type of study will be rated. In the end, MMAT scores will vary from $0 \%$ (zero criteria met, poor quality) to $100 \%$ (all criteria met, high quality). Consensus will be reached for each article's final scores and will be validated by the principal author (JGL). Also, it should be mentioned that the risk of bias of each study will be summarised as a narrative statement.

\section{Data synthesis}

As previously mentioned, the articles will be analysed according to their respective designs (ie, quantitative, qualitative or mixed-methods) as well as by their rating score according to the five criteria presented in the MMAT to evaluate their quality. This synthesis will be performed 
by two authors (authors SB and JF) and reviewed and validated by the principal author (JGL).

A descriptive and narrative synthesis of the findings will be presented using the information gathered in the evidentiary table, describing the effectiveness of available transition programmes in terms of quantitative data (eg, mean time to transfer, measures of disease control, proportion of patients transferred, percentage of mortality, frequency of emergency room use) or qualitative data (eg, views, experiences, perceptions) as well as consistency and discrepancies between studies. Risk of bias assessments will be incorporated into the synthesis as well as predictors, factors and determinants involved in the transition process, if any. A meta-analysis will not be possible given that the studies will most probably differ significantly in regard to design, purpose and outcomes.

\section{Amendments}

Any amendments to this protocol will be documented with references to saved searches and analysis methods, which will be recorded in the bibliographic databases and evidentiary table for data collection and synthesis.

\section{Ethics and dissemination}

Ethical approval for this systematic literature review will not be undertaken because the data to be collected cannot be linked to individuals.

Planned dissemination will primarily take the form of submissions for publication in recognised research journals. Other means of dissemination may include presenting the findings to partners, stakeholders and decision makers through research reports and at conferences. Furthermore, once the state of knowledge regarding this issue is established through this systematic literature review, the data will contribute to a research project with the aim of modelling a transition process in care and in life for ABI youth and their families, which will be published once it is completed.

\section{Author affiliations}

${ }^{1}$ Faculty of Nursing, Université de Montréal, Montréal, Québec, Canada

${ }^{2}$ Research Center of the Sainte-Justine University Hospital, Montréal, Québec, Canada

${ }^{3}$ Surgical-Traumatology \& Pediatric Burn Unit, Sainte-Justine University Hospital, Montréal, Québec, Canada

${ }^{4}$ Nursing Administration, Centre Hospitalier de I'Université de Montréal, Montréal, Québec, Canada

${ }^{5}$ Department of Paediatrics, Montreal Children's Hospital of the McGill University Health Centre, Montréal, Québec, Canada

${ }^{6}$ Department of Paediatrics, Marie Enfant Rehabilitation Centre, Sainte-Justine University Hospital, Montréal, Québec, Canada

${ }^{7}$ Neuromuscular Diseases, Marie Enfant Rehabilitation Centre, Sainte-Justine University Hospital, Montréal, Québec, Canada

Acknowledgements The authors would like to thank Fannie Tremblay-Racine from Sainte-Justine University Health Centre for her assistance in developing the search strategies.

Contributors JF led the development of the protocol, wrote the first draft and coordinated and integrated comments from coauthors. SB critically reviewed successive drafts of the manuscript and provided important intellectual input throughout the development of this protocol. JG-L conceived the study, provided supervision and mentorship to coauthors, critically reviewed successive drafts of the manuscript and provided important intellectual input. LK, MJ, JL and ASSC critically reviewed the final draft. JG-L approved the final version for publication and is the guarantor of the manuscript.

Funding The authors have not declared a specific grant for this research from any funding agency in the public, commercial or not-for-profit sectors.

Competing interests None declared.

Patient consent for publication Not required.

Provenance and peer review Not commissioned; externally peer reviewed.

Open access This is an open access article distributed in accordance with the Creative Commons Attribution Non Commercial (CC BY-NC 4.0) license, which permits others to distribute, remix, adapt, build upon this work non-commercially, and license their derivative works on different terms, provided the original work is properly cited, appropriate credit is given, any changes made indicated, and the use is non-commercial. See: http://creativecommons.org/licenses/by-nc/4.0/.

ORCID iD

Jérôme Gauvin-Lepage http://orcid.org/0000-0002-9852-2522

\section{REFERENCES}

1 Pearse RS, McConachie H, Colver AF. Transition of young people with long term conditions: the difference between intended provision and actual experience. Dev med Child Neurol Suppl 2016;58:21.

2 Rosen DS, Blum RW, Britto M, et al. Transition to adult health care for adolescents and young adults with chronic conditions: position paper of the Society for adolescent medicine. J Adolesc Health 2003;33:309-11.

3 Zhou H, Roberts P, Dhaliwal S, et al. Transitioning adolescent and young adults with chronic disease and/or disabilities from paediatric to adult care services - an integrative review. J Clin Nurs 2016;25:3113-30.

4 Department of Health Western Australia. Paediatric chronic disease transition framework. Perth, WA: Health Networks Branch, Department of Health Western Australia, 2009.

5 Gilliam PP, Ellen JM, Leonard L, et al. Transition of adolescents with HIV to adult care: characteristics and current practices of the adolescent trials network for HIV/AIDS interventions. J Assoc Nurses AIDS Care 2011;22:283-94.

6 Stewart D, Law M, Young NL, et al. Complexities during transitions to adulthood for youth with disabilities: person-environment interactions. Disabil Rehabil 2014;36:1998-2004.

7 Westwood A, Langerak N, Fieggen G. Transition from child- to adult-orientated care for children with long-term health conditions: a process, not an event. S Afr Med J 2014;104:310-3.

8 Milnes L. Coordinated and individualised transition planning for young people with complex needs. Nurs Child Young People 2016;28:19.

9 Viner RM. Transition of care from paediatric to adult services: one part of improved health services for adolescents. Arch Dis Child 2008;93:160-3.

10 Davies H, Rennick J, Majnemer A. Transition from pediatric to adult health care for young adults with neurological disorders: parental perspectives. Can J Neurosci Nurs 2011;33:32-9.

11 Fegran L, Hall EOC, Uhrenfeldt L, et al. Adolescents' and young adults' transition experiences when transferring from paediatric to adult care: a qualitative metasynthesis. Int J Nurs Stud 2014:51:123-35.

12 de Montalembert M, Guitton C, French Reference Centre for Sickle Cell Disease. Transition from paediatric to adult care for patients with sickle cell disease. Br J Haematol 2014;164:630-5.

13 Valenzuela JM, Buchanan CL, Radcliffe J, et al. Transition to adult services among behaviorally infected adolescents with HIV--a qualitative study. J Pediatr Psychol 2011;36:134-40.

14 Hanna KM, Woodward J. The transition from pediatric to adult diabetes care services. Clin Nurse Spec 2013;27:132-45.

15 Huang JS, Terrones L, Tompane T, et al. Preparing adolescents with chronic disease for transition to adult care: a technology program. Pediatrics 2014;133:e1639-46.

16 de Silva PSA, Fishman LN. Transition of the patient with IBD from pediatric to adult care-an assessment of current evidence. Inflamm Bowel Dis 2014;20:1458-64.

17 Bjornson K, Kobayashi A, Zhou C, et al. Relationship of therapy to postsecondary education and employment in young adults with physical disabilities. Pediatr Phys Ther 2011;23:179-86. 
18 Chamberlain MA, Kent RM. The needs of young people with disabilities in transition from paediatric to adult services. Eura Medicophys 2005;41:111-23.

19 Coyne I, Ferris ME. Transition from child to adult services: current research, theory and practice. J Pediatr Nurs 2015;30:635-7.

20 van Staa AL, Jedeloo S, van Meeteren J, et al. Crossing the transition chasm: experiences and recommendations for improving transitional care of young adults, parents and providers. Child Care Health Dev 2011;37:821-32.

21 Kingsnorth S, Healy H, Macarthur C. Preparing for adulthood: a systematic review of life skill programs for youth with physical disabilities. J Adolesc Health 2007;41:323-32.

22 Crowley R, Wolfe I, Lock K, et al. Improving the transition between paediatric and adult healthcare: a systematic review. Arch Dis Child 2011:96:548-53.

23 Grant C, Pan J. A comparison of five transition programmes for youth with chronic illness in Canada. Child Care Health Dev 2011;37:815-20.

24 Chaudhry SR, Keaton M, Nasr SZ. Evaluation of a cystic fibrosis transition program from pediatric to adult care. Pediatr Pulmonol 2013;48:658-65.

25 Hart LC, Patel-Nguyen SV, Merkley MG, et al. An evidence map for interventions addressing transition from pediatric to adult care: a systematic review of systematic reviews. J Pediatr Nurs 2019:48:18-34

26 Got Transition. Resources for providers, 2014. Available: http://www. gottransition.org/providers/index.cfm

27 Doug M, Adi Y, Williams J, et al. Transition to adult services for children and young people with palliative care needs: a systematic review. Arch Dis Child 2011;96:78-84.

28 Hankins JS, Osarogiagbon R, Adams-Graves P, et al. A transition pilot program for adolescents with sickle cell disease. J Pediatr Health Care 2012;26:e45-9.

29 Moher D, Shamseer L, Clarke M, et al. Preferred reporting items for systematic review and meta-analysis protocols (PRISMA-P) 2015 statement. Syst Rev 2015;4:1.

30 de Jongh T, Gurol-Urganci I, Vodopivec-Jamsek V, et al. Mobile phone messaging for facilitating self-management of long-term illnesses. Cochrane Database Syst Rev 2012;5.

31 Hong QN, Fàbregues S, Bartlett G, et al. The mixed methods appraisal tool (MMAT) version 2018 for information professionals and researchers. Education for Information 2018;34:285-91.

32 National Collaborating Centre for Methods and Tools. Appraising qualitative, quantitative and mixed methods studies included in mixed studies reviews: the MMAT. Hamilton, ON: McMaster University, 2017: $1-3$. 